\title{
Estudio de las alteraciones de la agregación eritrocitaria en pacientes con accidente cerebrovascular isquémico.
}

\author{
Study of erythrocyte aggregation alterations in patients \\ with ischemic stroke.
}

\author{
Jorge Capriotti Corrente ${ }^{1}$, María J. Svetaz ${ }^{3}$, Leda Urli², Elsa Raimondi ${ }^{1}$, Alejandra Luquita ${ }^{2}$. \\ ${ }^{1}$ Servicio de Neurología. Hospital Provincial del Centenario. Rosario; Argentina. \\ ${ }^{2}$ Facultad Ciencias Médicas. Universidad Nacional de Rosario. Rosario; Argentina. \\ ${ }^{3}$ Facultad Ciencias Bioquímicas y Farmacéuticas. Universidad Nacional de Rosario. Rosario; Argentina.
}

Autor por correspondencia: Jorge Capriotti Corrente — jorgepcapriotti@gmail.com Conflicto de intereses: no presenta.

\section{Resumen}

El accidente cerebrovascular (ACV) isquémico es causado por evolución de procesos aterotrombóticos que se relacionan con daño y disfunción endotelial debido a patologías crónicas como hipertensión arterial y dislipemia.

En trabajos previos se ha informado sobre alteraciones hemorreológicas en el curso de la patología vascular cerebral, en particular el incremento en la agregación eritrocitaria (AE).

El presente trabajo tiene como objetivo principal estudiar las modificaciones de las propiedades hemorreológicas y en particular de la AE, en 20 pacientes con ACV isquémico durante la etapa aguda, en comparación a un grupo control $(n=20)$ con similares factores de riesgo.

Se determinó en plasma: concentración de fibrinógeno, proteína C reactiva y triglicéridos. En sangre: agregación eritrocitaria por densitometría óptica, obteniéndose el tamaño promedio de los agregados y la velocidad inicial del proceso, velocidad de eritrosedimentación, índice de rigidez eritrocitaria, viscosidad sanguínea, viscosidad plasmática y viscosidad sanguínea relativa estandarizada a un hematocrito del $45 \%$.

Se encontró que los eritrocitos de los pacientes con ACV agregaron a mayor velocidad (0,92 \pm $0,64$ vs 0,49 $\pm 0,24 ; p<0,00001)$ y formaron agregados de mayor tamaño $(1,84 \pm 0,07$ vs 1,81 $\pm 0,07$; $\mathrm{p}<0,05)$ que los controles. Este fenómeno correlacionó ( $\mathrm{r}$ : coeficiente de Pearson) con las alteraciones encontradas en el plasma, en particular con las concentraciones séricas de fibrinógeno $(r=0,565 ; p$ $<0,01)$ y triglicéridos $(r=0,693 ; p<0,001)$. La identificación de estos factores es de relevancia clínica ya que podría tener incidencia en las estrategias terapéuticas.

Palabras clave: Accidente cerebrovascular. Agregación eritrocitaria. Fibrinógeno. Triglicéridos.

\begin{abstract}
Isquemic stroke is the result of the evolution of atherothrombotic processes that are related to endothelial damage and dysfunction cause by chronic diseases such as hypertension and dyslipemia.

Previous works has been reported about hemorheological disturbances in the course of cerebrovascular diseases, including increased erythrocyte aggregation (EA).
\end{abstract}


The main objective of this study is to evaluate the changes in hemorrheological properties and in particular the erythrocyte aggregation process, which occur in 20 patients with ischemic stroke during the acute stage, compared to a control group $(\mathrm{n}=20)$ with similar risk factors.

Concentration of fibrinogen $(\mathrm{Fb}), \mathrm{C}$ reactive protein and triglycerides $(\mathrm{TG})$ were determined in plasma. Into blood: EA, by optical densitometry, getting the average size of the aggregates and the initial speed of the process, erythrocyte sedimentation rate, erythrocyte rigidity index, blood viscosity, plasma viscosity and standardized relative blood viscosity at $45 \%$ hematocrit.

We found that the erythrocytes of patients with stroke aggregate faster $(0,92 \pm 0,49$ vs 0,64 $\pm 0,24, \mathrm{p}<0,00001)$ and form larger aggregates $(1,84 \pm 0,07 \mathrm{vs} 1,81 \pm 0,07, \mathrm{p}<0,05)$ than controls. This phenomenon correlated ( $\mathrm{r}$ : Pearson's coefficient) with plasma alterations, particularly with $\mathrm{Fb}(\mathrm{r}=$ $0,565, p<0,01)$ and $T G(r=0,693, p<0,001)$. Identification of these factors is clinically relevant since it could have an impact on therapeutic strategies.

Keywords: Isquemic stroke. Erythrocyte aggregation. Fibrinogen. Triglycerides.

\section{Introducción}

El accidente cerebrovascular (ACV) o ictus de origen isquémico es causado en la mayoría de los casos por la evolución de procesos aterotrombóticos, relacionados a un estado inflamatorio crónico y disfunción endotelial, en patologías crónicas como hipertensión arterial, diabetes mellitus, tabaquismo y dislipemia (1-3). Entre los mencionados factores de riesgo clásicos, las alteraciones en las propiedades del flujo sanguíneo han demostrado desempeñar un rol crucial en el desarrollo y evolución de la patología cerebrovascular (4-6).

Sedenomina hemorreología al estudio delas propiedades deflujoy deformación delos componentes celulares y plasmáticos de la sangre, y su interacción con la pared de los vasos sanguíneos (7). La condición de accidente cerebrovascular agudo se acompaña de perturbaciones en los parámetros hemorreológicos, que pueden ser determinados en la circulación periférica $(8,9)$. Los eritrocitos pueden incrementar su agregación, es decir su tendencia a formar rouleaux o pilas de monedas, lo cual conduce a un incremento de la viscosidad sanguínea con la indudable consecuencia de un aumento de la resistencia al flujo sanguíneo. Esta situación se observa en diferentes condiciones asociadas fundamentalmente a procesos inflamatorios (10,11), así como en el ACV (12). Un incremento de la agregación eritrocitaria ( $\mathrm{AE}$ ) contribuiría a los desórdenes circulatorios, y especialmente en la microcirculación a la oclusión de los capilares $(4,13,14)$. La presencia de macromoléculas en el plasma, cuyo efecto es formar puentes, es una condición necesaria para que la agregación de los eritrocitos tenga lugar. En el plasma, el fibrinógeno (Fb) es la principal proteína

pro-agregante, aunque también coadyuvan las globulinas y otras proteínas de fase aguda, así como también cambios en los niveles plasmáticos de las distintas fracciones lipídicas; por el contrario, la albúmina desfavorece el fenómeno de agregación $(15,16)$. El fibrinógeno como la principal proteína pro-agregante es un marcador del proceso inflamatorio y del deterioro de la función endotelial, y por lo tanto se lo puede considerar como un predictor de enfermedad cardiovascular; en particular de ACV y de mortalidad, en personas sanas de mediana edad (17). Estudios realizados en pacientes obesos y fumadores que presentaban aumento de fibrinógeno y de proteínas inflamatorias, pusieron en evidencia la precedente asociación (18).

En la actualidad el rol que desempeñan los lípidos en la AE es controversial. El aumento de la AE encontrado en pacientes dislipémicos podría deberse a una mayor producción hepática de $\mathrm{Fb}$, a modificaciones en la composición lipídica de la membrana celular (19), o a cambios en los niveles plasmáticos de las distintas fracciones lipídicas (20).

En definitiva, no se ha determinado de manera experimental cómo los factores celulares y plasmáticos interaccionan para modificar la velocidad y el tamaño de los agregados eritrocitarios durante el ACV y si ésta es una relación causa-efecto. El presente trabajo tiene como objetivo principal estudiar las modificaciones de las propiedades hemorreológicas y en particular la velocidad inicial del proceso de agregación eritrocitaria y el tamaño de los agregados, que ocurren en los pacientes con ACV isquémico durante la etapa aguda, en comparación a un grupo control con presencia de similares factores de riesgo y sin alteraciones neurológicas. 


\section{Materiales y métodos}

Se realizó un estudio descriptivo, de tipo observacional y transversal. La población de estudio fueron los pacientes con diagnóstico de ACV isquémico ingresados en el Hospital Provincial del Centenario de la ciudad de Rosario, Santa Fe, Argentina, durante el período comprendido entre mayo de 2011 y abril de 2012. El Comité de Ética del hospital aprobó el protocolo de estudio (Expediente 2623/2010). Todos los participantes firmaron un consentimiento informado de acuerdo con las recomendaciones de la Declaración de Helsinki (21), o en caso de impedimentos lo hizo un familiar.

Se incluyeron en el estudio 20 pacientes con afección neurológica focal de aparición súbita, que perduró más de 24 horas y de presunto origen vascular (definición normalizada de la OMS para ACV) que consultaron en el servicio de guardia del hospital. El diagnóstico fue determinado por el examen clínico general y neurológico, pruebas de laboratorio y neuroimagen (cambios sugestivos de infarto cerebral agudo en Tomografía Axial Computada realizada al ingreso y a las 24hs). Los criterios de exclusión al estudio fueron: tiempo de comienzo de los síntomas mayor a 72 horas antes de la consulta, accidente isquémico transitorio, tratamiento trombolítico o anticoagulante, síntomas neurológicos causados por trauma de cráneo o tóxicos, hemorragia intraparenquimatosa, hemorragia subdural, hemorragia epidural, alteraciones conocidas de la coagulación-hemostasia, anemia severa, insuficiencia renal aguda o crónica, insuficiencia hepática, cáncer, serología positiva para el virus de inmunodeficiencia humana (HIV). La administración previa de antiagregantes plaquetarios (ácido acetil-salicílico, clopidogrel) o bajas dosis de heparina profiláctica no fueron criterios de exclusión del estudio y se consignó en las fichas individuales.

El grupo control correspondió a 20 sujetos seleccionados por la presencia de factores de riesgo clásicos para $\mathrm{ACV}$, sin síntomas neurológicos actuales, que fueron captados durante la realización de charlas a la comunidad sobre la prevención de enfermedad cerebrovascular. Se excluyeron del grupo control sujetos con enfermedades clínicamente significativas detectables por los antecedentes y el examen físico. Posteriormente, se completaron las fichas con los datos personales, y se realizaron las determinaciones bioquímicas y hemorreológicas.

Determinaciones bioquímicas: colesterol total por método enzimático de esterasa-oxidasa, colesterol HDL y LDL por método colorimétrico utilizando Fenol/4-aminofenazona y triglicéridos (TG) por método enzimático con los reactivos lipoprotein lipasa, glicerol kinasa, glicerol fosfato oxidasa, peroxidasa, adenosina trifosfato y 4-aminofenazona (utilizando en todas las determinaciones equipos del Laboratorio Wiener). Hematocrito (Hto) por micrométodo. Contaje de glóbulos rojos con cámara de Newbauer. Volumen corpuscular medio (VCM) por cálculo a partir del hematocrito y el número de glóbulos rojos. Concentración de hemoglobina corpuscular media (CHbCM) a partir del hematocrito y de la concentración de hemoglobina $(\mathrm{Hb})$ por método espectrofotométrico (método de la cianmetahemoglobina) (22). Dosaje de fibrinógeno por método de Clauss, método de referencia del Comité Nacional de Estándares de Laboratorio Clínico. Proteína C reactiva por la técnica de Singer y Plotz. Velocidad de eritrosedimentación por método Westergreen.

Determinaciones hemorreológicas: viscosidad sanguínea (VS) y plasmática (VP) se determinaron en sangre entera de pacientes y controles al hematocrito nativo, y en el plasma separado por centrifugación a 5000 revoluciones por minuto (RPM) con un viscosímetro rotacional cono-plato Wells-Brookfield LVT (Brookfield, Stoughton, MA, USA) a una velocidad de corte de 230 $\operatorname{seg}^{-1} \mathrm{y}$ temperatura de $37^{\circ} \mathrm{C}$.

La viscosidad sanguínea relativa (VR) se calculó a un hematocrito estándar del 45\% y para ello se utilizó la ecuación VR = (VS/VP) ${ }^{45 / \text { Hto }}$ para evitar así la influencia directa tanto de la viscosidad plasmática como del porcentaje de glóbulos rojos en las mediciones (23).

El índice de rigidez (IR), inversa de la deformabilidad eritrocitaria, se midió por el método de filtración (24) por membranas de policarbonato en un aparato automatizado. Con este método, la sangre entera de pacientes y controles se centrifugó a 5000 RPM durante 5 minutos, el plasma y la capa leucocitaria fueron separados y los eritrocitos se lavaron dos veces con buffer fosfato isotónico. Los glóbulos rojos lavados $(1 \mathrm{ml})$ se volvieron a suspender en buffer fosfato isotónico con albúmina bovina (9 ml) (Sigma Chemical Co., St.Louis, MO, EE. UU.) para conservar la forma del eritrocito, obteniendo un hematocrito del $10 \%$.

Luego, se pasó la suspensión de eritrocitos a través de un filtro de policarbonato, con 5 micrometros de tamaño de poro (Nucleopore Corp. USA), usando una presión de filtración negativa de $10 \mathrm{~cm} \mathrm{H}_{2} \mathrm{O}$. 
El tiempo de flujo requerido para que un $1 \mathrm{ml}$ de suspensión de eritrocitos pase a través del filtro se midió en pacientes y controles. Los resultados se expresaron como un índice de rigidez (IR) que es una estimación de la deformabilidad eritrocitaria (25), definido como:

$\mathrm{IR}=(\mathrm{Tb}-\mathrm{Ts}) /(\mathrm{Ts}) \times 100 /$ Hto

Donde:

Tb: tiempo de paso de la suspensión celular a través del filtro; Ts: tiempo de paso de un volumen igual de buffer fosfato isotónico; Hto: hematocrito (10\%).

Las medidas de deformabilidad de eritrocitos están en conformidad con el Comité Internacional de Normalización en Hematología (26).

Agregación eritrocitaria (AE): se realizaron las mediciones con un agregómetro, instrumento que consta de un sistema mecánico que agita la muestra de sangre a una velocidad media controlada de 560 seg-1 $^{-1}$ para obtener una desagregación completa. Luego de una abrupta interrupción de la agitación, el circuito de medición verifica por medio del receptor infrarrojo una gradual disminución en la transmitancia de la luz asociado con la producción de agregados de células rojas. Esta señal es luego amplificada y conectada a la entrada del conversor analógico-digital de la placa de adquisición de datos. Posteriormente, el software los grafica en línea, permitiendo al operador observar a cada instante lo que está sucediendo, en forma cuantitativa y gráfica. Del análisis de la curva se determinan dos parámetros del proceso de AE: $\mathrm{S}_{0} \mathrm{~N}_{0}$ que estima el tamaño promedio de los agregados $(\mathrm{T})$, y $2 \mathrm{~K}_{2} \mathrm{~N}_{0}$ que estima la velocidad inicial del proceso (V) $(27,28)$.

Las muestras de sangre se obtuvieron por extracción venosa de pacientes y controles, fueron anticoaguladas con EDTA (Sigma, Chem Co) y se usaron dentro de las cuatro horas.

Los glóbulos rojos de ambos grupos, obtenidos por centrifugación a 5000 RPM, fueron resuspendidos al 40\% de hematocrito, en su propio plasma y en dextrán 500 al 2\% de la solución buffer fosfato isotónico (Osmolaridad 290 mOsm, pH 7,4), esto último sirve para valorar el efecto celular de los eritrocitos sobre la agregación favorecida por dextrán como molécula agregante, independientemente de las proteínas presentes en el plasma (29).

El análisis estadístico se realizó con el software SPSS. La prevalencia de las variables cualitativas se presentó como frecuencias absolutas y porcentaje mientras que los datos de las determinaciones bioquímicas y hemorreológicas se presentaron como media \pm desvío standard.

Las comparaciones entre grupos fueron analizadas aplicando la t de Student para grupos no apareados. La correlación entre los parámetros hemorreológicos (viscosidad sanguínea relativa, velocidad de los agregados eritrocitarios) y velocidad de eritrosedimentación, concentraciones séricas de fibrinógeno y triglicéridos en pacientes con ACV, se calculó mediante el coeficiente de correlación de Pearson. Se consideró diferencia estadística sólo cuando p fue menor a 0,05 $(p<0,05)$

\section{Resultados}

En la Tabla 1 se muestran las características clínicas y demográficas de los 20 pacientes con diagnóstico de ACV isquémico y los 20 sujetos controles que se incluyeron en el estudio. En los pacientes los principales factores de riesgo para ACV encontrados fueron hipertensión arterial (91\%, 19/20), tabaquismo (80\%, 16/20) y dislipemia (70\%, 14/20), en el 40\% (8/20) de los pacientes coexistían 3 factores de riesgo. 
Tabla 1. Características clínicas en controles y pacientes con ACV isquémico.

\begin{tabular}{|l|c|c|c|}
\hline Parámetros & Controles $(\mathrm{n}=20)$ & Pacientes con ACV $(\mathrm{n}=20)$ & Nivel significado \\
\hline Mujeres/hombres & $13 / 7$ & $4 / 16$ & - \\
\hline Edad (años) & $67,7 \pm 9$ & $57,7 \pm 10$ & $\mathrm{p}<0,01$ \\
\hline Perímetro abdominal (cm) & $103 \pm 14$ & $102 \pm 10$ & $\mathrm{p}=0,83$ \\
\hline Fumadores & 0 & 16 & $\mathrm{p}<0,01$ \\
\hline Hipertensión arterial & 13 & 19 & $\mathrm{p}=0,027$ \\
\hline Diabetes Mellitus & 1 & 4 & $\mathrm{p}=0,07$ \\
\hline Colesterol (mg/dl) & $231 \pm 13,3$ & $178,2 \pm 11,6$ & $\mathrm{p}<0,01$ \\
\hline Triglicéridos (mg/dl & $152,2 \pm 17,9$ & $156,3 \pm 19,9$ & $\mathrm{p}=0,88$ \\
\hline HDL (mg/dl) & $61,9 \pm 4,1$ & $37,1 \pm 2,1$ & $\mathrm{p}<0,001$ \\
\hline LDL (mg/dl) & $136,8 \pm 11,9$ & $109,7 \pm 9,7$ & $\mathrm{p}=0,08$ \\
\hline
\end{tabular}

Como se observa en la Tabla 2, los valores de VCM, CHbCM, PCR y Fb resultaron elevados significativamente en los pacientes con ACV en comparación a los valores controles. Los pacientes con ACV isquémico mostraron un leve aumento del índice de rigidez y de la viscosidad sanguínea relativa respecto a los controles, sin resultar estadísticamente significativos.

Tabla 2. Variables bioquímicas y hemorreológicas en controles y pacientes con ACV.

\begin{tabular}{|c|c|c|c|}
\hline Variables & Controles & Pacientes & Nivel significado \\
\hline Fibrinógeno (g/l) & $2,8 \pm 0,2$ & $4,01 \pm 0,4$ & $\mathrm{p}<0,01$ \\
\hline PCR (mg/l) & $1,5 \pm 0,4$ & $12,1 \pm 3,1$ & $\mathrm{p}<0,001$ \\
\hline CHbCM (g/dl) & $32,5 \pm 0,8$ & $33,6 \pm 1,5$ & $\mathrm{p}<0,01$ \\
\hline $\operatorname{VCM}(\mathrm{fl})$ & $86,1 \pm 7,1$ & $90,8 \pm 3,7$ & $\mathrm{p}<0,01$ \\
\hline IR & $6,9 \pm 1,3$ & $8,7 \pm 2,4$ & $\mathrm{p}=0,075$ \\
\hline $\mathrm{VP}(\mathrm{cP})$ & $1,5 \pm 0,4$ & $1,5 \pm 0,2$ & $\mathrm{p}=0,153$ \\
\hline VR (cP) & $3,2 \pm 0,4$ & $3,5 \pm 0,6$ & $\mathrm{p}=0,058$ \\
\hline \multicolumn{4}{|c|}{$\begin{array}{l}\text { PCR: proteína C reactiva. CHbCM: concentración de hemoglobina corpuscular media. VCM: volumen corpuscular medio. IR: índice de } \\
\text { rigidez. VP: viscosidad plasmática a una velocidad de corte de } 230 \mathrm{seg}^{-1} \text {.VR: viscosidad sanguínea relativa estandarizada a un hematocrito de } \\
45 \% \text { y una velocidad de corte de } 230 \mathrm{seg}^{-1} \text {. }\end{array}$} \\
\hline
\end{tabular}

Como se observa en las Figuras 1 y 2, los eritrocitos de los pacientes con ACV isquémico agregaron a mayor velocidad, y formaron agregados de mayor tamaño que en los controles. Esto ocurrió tanto en las muestras de sangre entera como en las muestras de los eritrocitos lavados suspendidos en dextrán 500 al $2 \%$ de pacientes y controles. 


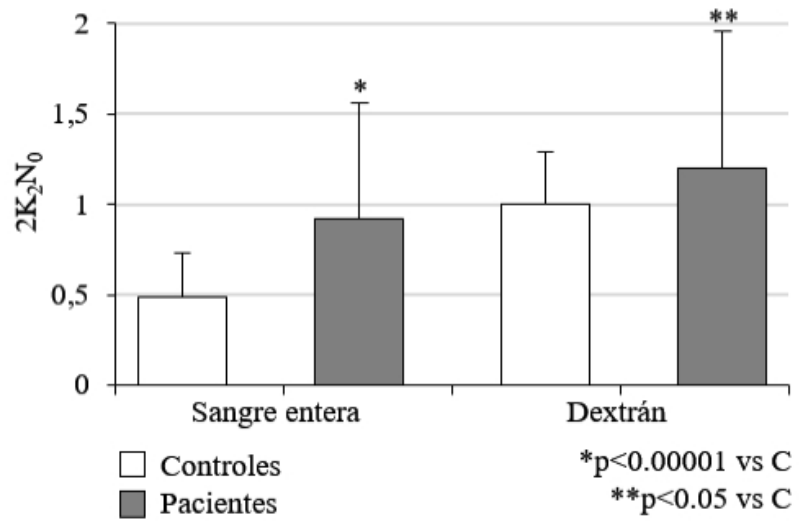

$2 \mathrm{~K}_{2} \mathrm{No}$ : velocidad de agregación eritrocitaria

Figura 1: Comparación de velocidad de agregación eritrocitaria en sangre entera y en dextrán en pacientes con ACV vs. controles.

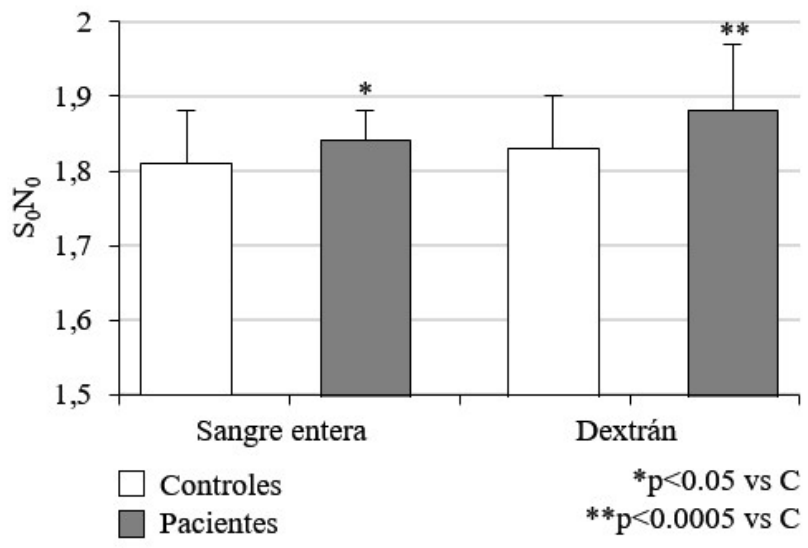

SoNo: tamaño de los agregados eritrocitarios.

Figura 2: Comparación del tamaño de los agregados eritrocitaria en sangre entera y en dextrán en pacientes con ACV vs. controles.

La Tabla 3 muestra los coeficientes de correlación de Pearson entre los parámetros hemorreológicos (viscosidad sanguínea relativa, velocidad de los agregados eritrocitarios) y velocidad de eritrosedimentación, concentraciones séricas de fibrinógeno y triglicéridos en pacientes con ACV. Las variables VSE y VR se correlacionaron positivamente con las concentraciones de fibrinógeno y triglicéridos, así como con la eritrosedimentación.

Tabla 3. Coeficientes de Correlación de Pearson (r) entre parámetros hemorreológicos.

\begin{tabular}{|l|c|c|c|}
\hline \multirow{2}{*}{ VSE } & Eritrosedimentación & Fibrinógeno & Triglicéridos \\
\hline \multirow{2}{*}{ VD } & $\mathrm{r}=0,90$ & $\mathrm{r}=0,565$ & $\mathrm{r}=0,693$ \\
\cline { 2 - 4 } & $\mathrm{p}<0,0001$ & $\mathrm{p}<0,01$ & $\mathrm{p}<0,001$ \\
\hline \multirow{2}{*}{ VR } & $\mathrm{r}=0,055$ & $\mathrm{r}=0,255$ & $\mathrm{p}=0,165$ \\
\cline { 2 - 4 } & $\mathrm{p}=0,153$ & $\mathrm{r}=0,690$ & $\mathrm{r}=0,56$ \\
\hline
\end{tabular}




\section{Discusión}

En el presente trabajo se estudió la velocidad inicial del proceso de agregación eritrocitaria y el tamaño de los agregados en pacientes con ACV isquémico en comparación a un grupo control, así como los factores celulares y plasmáticos que influencian la AE.

En concordancia con otros estudios $(14,30,31)$ fue demostrado en los pacientes con ACV, un incremento en la agregación eritrocitaria, respecto de los controles.

Las concentraciones de proteína $\mathrm{C}$ reactiva y fibrinógeno se encontraron incrementadas significativamente en los pacientes con respecto a los controles, como se observa en otros procesos inflamatorios (10-12). Se ha demostrado que el Fb induce agregación, ya sea por un mecanismo de uniones no específicas como por su estructura molecular, apropiada para formar puentes intercelulares en la superficie de los eritrocitos $(15,16)$. Estos resultados sugieren un rol predominante del plasma en la agregación eritrocitaria incrementada de los pacientes con ACV. Varios componentes podrían tener un papel relevante en este proceso.

Se ha sugerido que la dislipemia podría ser uno de los fenómenos primarios relacionados al aumento de la agregación y éste el mecanismo fisiopatológico por el cual se incrementa el riesgo cardiovascular en el síndrome metabólico (32). Además, se ha destacado la mayor relevancia de la concentración de triglicéridos por sobre colesterol o LDL en los mecanismos que desencadenan la enfermedad vascular (20). En coincidencia con éstas referencias, hallamos en nuestros pacientes con $\mathrm{ACV}$ isquémico una correlación entre la velocidad de AE en sangre entera y triglicéridos $(p<0.001)$ que no se observó con otras fracciones lipídicas como colesterol total o LDL, a pesar de no presentar diferencias significativas en las concentraciones plasmáticas de TG de ambos grupos.

Entre los factores celulares estudiados que podrían afectar el proceso de agregación, encontramos que el volumen corpuscular medio del eritrocito y la concentración de hemoglobina corpuscular media mostraron diferencias significativas con los controles. Este incremento del volumen globular disminuye la relación superficie/ volumen globular. Además, la incrementada concentración de hemoglobina corpuscular media estima un aumento de la viscosidad interna del eritrocito (33).

Llamativamente, no encontramos diferencias significativas en la viscosidad plasmática de ambos grupos, medida a altas velocidades de corte, lo cual coincide con los resultados hallados en estudios previos de pacientes hipertensos (34).

Diferentes investigaciones han informado que alteraciones pronunciadas de la agregación eritrocitaria en pacientes con enfermedad cerebrovascular fueron relacionadas a condiciones patológicas como hipertensión arterial, diabetes, dislipemia, y se sugirió que controlando mejor los niveles de glucosa o la presión arterial podría reducirse la AE en estos pacientes, y afectar el curso clínico de la enfermedad $(35,36)$. Con respecto a los factores de riesgo relevados en nuestro estudio, la hipertensión arterial fue el más frecuente en ambos grupos, 91\% de los pacientes con ACV y 72,2\% de los controles, seguido por obesidad, dislipemia y en menor proporción diabetes. El tabaquismo representó un factor de riesgo prevalente en el grupo de pacientes con ACV.

En síntesis, nuestro estudio con un pequeño número de pacientes encontró alteraciones de la reología eritrocitaria en el curso agudo de la patología vascular cerebraly, en particular, un incremento en la agregación eritrocitaria. Las concentraciones elevadas de Fb en plasma de los pacientes con ACV isquémico podría ser un factor relevante para el incremento de la $\mathrm{AE}$ y relacionarse a un estado proinflamatorio y a los factores de riesgo clásicos. El rol de otros factores plasmáticos (37) y celulares que influyen en el complejo proceso de agregación aún resta ser dilucidado y requerirá de mayores estudios.

Desde el punto de vista clínico proponemos que tanto la agregación eritrocitaria como la concentración de fibrinógeno podrían monitorearse rutinariamente en los sujetos con los factores de riesgo mencionados, ya que pueden ser el objetivo de medidas preventivas y terapéuticas relevantes.

Agradecimientos: al Ministerio de Salud de la Nación por la Beca Carrillo - Oñativia otorgada para el financiamiento de esta investigación. Al servicio de Neurología del Hospital Centenario y a la Dra. Elsa Raimondi por su estímulo y colaboración permanente. A la Lic. Teresa Bisso por su valioso aporte en estadística.

Fuente de financiamiento: no presenta. 


\section{Referencias bibliografícas}

1. Meschia J, Bushnell C, Boden-Albala B, et al. Guidelines for the Primary Prevention of Stroke. A Statement for Healthcare Professionals from the American Heart Association/American Stroke Association. Stroke. 2014 Dec; 45(12): $3754-3832$.

2. Who STEPS stroke manual. Manual de la OMS para la vigilancia paso a paso de accidentes cerebrovasculares. Enfermedades no Transmisibles y Salud Mental, Organización Mundial de la Salud. Actualización 2006.

3. Ionova VG. State of the rheological properties of blood in the dynamics of development of acute disturbances of cerebral circulation in atherosclerosis patients. J Eng Phys Thermophy 2003; 76: 672-680.

4. Tikhomirova IA, Oslyakova AO, Mikhailova SG. Microcirculation and blood rheology in patients with cerebrovascular disorders. Clin Hemorheol Microcirc 2011; 49: 295-305.

5. Toth A, Papp J, Rabai M, et al. The role of hemorheological factors in cardiovascular medicine. Clin Hemorheol Microcirc 2014; 56: 197-204.

6. Hashem S, Helmy S, El-Fayomy N, et al. Predictors of stroke outcome: the role of hemorheology, natural anticoagulants, and serum albumin. Egypt J Neurol Psychiatr Neurosurg. 2018; 54(1): 18.

7. 7-. Copley, AL. The history of Clinical Hemorheology. Clin Hemorheol Microcirc 1985; 5: 765-811.

8. McHedlishvili G, Shakarishvili R, Momtselidze N, et al. Comparative values of erythrocyte aggregability versus other indices of hemorheological disorders in patients with ischemic brain infarcts. Clin Hemorheol Microcirc 2000; 22(1): 9-15.

9. Marcinkowska-Gapinska A, Kowal P. Comparative analysis of chosen hemorheological methods in a group of stroke patients. Clin Hemorheol Microcirc 2009; 41(1): 27-33.

10. Rost NS, Wolf PA, Kase CS, et al. Plasma concentration of C-reactive protein and risk of ischemic stroke and transient ischemic attack: The Framingham study. Stroke 2001; 32(11): 2575-9.

11. Weng X, Cloutier G, Beaulieu R, et al. Influence of acute-phase proteins on erythrocyte aggregation. Am J Physiol Heart Circ Physiol 1996; 271: 2346-2352.

12. Woodward M, Lowe GD, Campbell DJ, et al. Associations of inflammatory and hemostatic variables with the risk of recurrent stroke. Stroke 2005; 36(10): 2143-7.

13. Ben Ami R, Barstein G, Zeltser D, et al. Parameters of red blood cell aggregation as correlates of the inflammatory state. Am.J.Physiol.Heart. Circ.Physiol 2001; 280:1982-8.

14. Kowal P, Marcinkowska-Gapinska A. Hemorheological changes dependent on the time from the onset of ischemic stroke. J Neurol Sci 2007; 258: 132-136.

15. Rampling NW, Whittinghstall P, Linderkamp O. The effects of fibrinogen and its plasmin degration products on the reology of erythrocyte suspensions. Clin Hemorheol Microcirc 1984; 4: 533-543.

16. Lominadaze D, Dean WL. Involvement of fibrinogen specific binding in erythrocyte aggregation. FEBS lett 2002; 517 : 41-44.

17. Chuang SY, Bai CH, Chen WH, et al. Fibrinogen independently predicts the development of ischemic stroke in a taiwanese population: CVDFACTS Study. Stroke 2009; 40:1578-1584.

18. Samocha-Bonet D, Lichtenberg D, Tomer A, et al. Enhanced erythrocyte adhesiveness/aggregation in obesity corresponds to low-grade inflammation. Obes Res 2003; 11: 403-407.

19. Vayd A, Martinez M, Carmena R, et al. Red blood cell aggregation and primary hyperlipoproteinemia. Thromb Res 1993; $72: 119-126$.

20. Gyawali P, Richards RS, Bwititi PT, et al. The association of dyslipidemia with erythrocyte aggregation. Clin Lipidol 2015; 10(2): 129-135

21. World Medical Association 52nd General Assembly. The International Response to Helsinki VI - The WMA's Declaration of Helsinki on Ethical Principles for Medical Research Involving Human Subjects. Edinburgh, Scotland, October 2000.

22. Van Kampen EJ, Zijlstra W. Standardization of hemoglobinometry. The hemoglobincyanide method. Clin Chim Acta 1961; 6: 538-544.

23. Chailley B, Giraud F, Claret M. Alteration in human erythrocyte shape and the state of spectrin and phospholipid phosphorilation induced by cholesterol depletion. Biochem Biophys Acta 1981; 643: 636-641.

24. Reid HL, Barnes AJ, Lock PJ, et al. A simple method for measuring erythocyte deformability. J Clin Pathol 1976; 29 : 855-858.

25. 25. Jones JG, Adams RA, Evans SA: Bulk filtration through micropore membranes for analysing blood cell rheology in clinical research. Clin Hemorheol 1994, 14:149-69.

26. 26. International Committee for Standardization in Haematology: Expert panel on blood rheology. Clin Hemorheol 1986, 6:439-52.

27. 27- Bollini A, Rasia M, Toro R, et al. Instrumento para la medición de agregación eritrocitaria. Rev Argent Bioingenieria 2000; 6: 54-70.

28. 28- Bertoluzzo SM, Bollini A, Rasia M, et al. Cynetic model of erythrocyte aggregation. Blood Cells Mol Dis 1999; 25 (22): 339-342.

29. 29- Neu B, Wenby R, Meiselman H. Effects of dextran molecular weight on red blood cell aggregation. Biophys J 2008; 95: 3059-3065.

30. 30-Velcheva I, Antonova N, Titianova E, et al. Hemorheological disturbances in cerebrovascular diseases. Clin Hemorheol Microcirc 2008; 39(1-4): 391-6. 
31. 31- Bolokadze N, Lobjanidze I, Momtselidze N, et al. Comparison of erythrocyte aggregability changes during ischemic and hemorrhagic stroke. Clin Hemorheol Microcirc 2006; 35 (1-2): 265-7.

32. 32- Gyawali P, Richards RS, Hughes DL, et al. Erythrocyte aggregation and metabolic syndrome. Clin Hemorheol Microcirc 2014; 57: 73-83.

33. 33- Gennaro AM, Luquita A, Rasia M. Comparison between internal microviscosity of low-density erythrocytes and the microviscosity of hemoglobin solutions: an electron paramagnetic resonance study. Biophys J 1996; 71(1): 389-93.

34. 34- Foresto P, D’arrigo M, Filippini F, et al. Evaluación de alteraciones hemorreológicas en pacientes hipertensos. Medicina (B. Aires) 2005; 65: 121-125.

35. 35-Szapary L, Horvath B, Marton Z, et al. Hemorheological disturbances in patients with chronic cerebrovascular diseases. Clin Hemorheol Microcirc 2004; 31(1): 1-9.

36. 36-Baskurt O, Neu B, Meiselman H. Red Blood Cell Aggregation. CRC Press 2011.

37. 37- Krüger-Genge A, Sternitzky R, Jung F, et al. Erythrocyte aggregation in relation to plasma proteins and lipids. J Cell Biotechnol 2019; 5: 65-70 